\title{
Separating the Effects of Hemodialysis Dose and Nutrition: In Search of the Optimal Dialysis Dose
}

\author{
Robert A. Wolfe and Friedrich K. Port \\ United States Renal Data System Coordinating Center and University of Michigan, Ann Arbor, Michigan
}

\begin{abstract}
The renal community owes Dr. Frank Gotch a great debt for the contributions that he has made to clarify the relationship between urea clearance and the dose of dialysis. With John Sargent he developed the mathematical approach to hemodialysis (1). In his seminal publication he re-analyzed data of the National Cooperative Dialysis Study to establish the association between the theoretically derived $K t / V$ for urea and clinical outcomes (2). Although diffusion equations are well understood and are used in many fields of science, Dr. Gotch's persistent and tireless efforts to identify and quantitate the specific aspects of this process in dialysis through formal kinetic modeling, yielding additionally the protein catabolic rate $(P C R)(3)$, have been extremely valuable to both patients and clinicians alike. The continuing measurement of Kt/ $V_{\text {urea }}$ in every dialysis unit is a lasting tribute to $\mathrm{Dr}$. Gotch's contributions.

Accurate measurement of the dose of dialysis is important in part because of its relationship to patient outcomes and to the cost of dialysis. Further, the concepts underlying the dose of episodic hemodialysis, pioneered by Dr. Gotch, are of practical importance in understanding the role of residual renal function and in quantitateing the dose of continuous dialytic procedures. Kt/V has been found to be one of the key modifiable components of the practice of hemodialysis.
\end{abstract}

Several recent presentations have identified nutritional factors as among the strongest predictors of end-stage renal disease (ESRD) patient mortality (4-6). There is no single omnibus measure of patient nutritional status, but several surrogates, including clinical assessment, higher serum creatinine, albumin, prealbumin, patient weight (volume or total body water), body mass index (BMI), and urea generation (or PCR), have been shown to be strong correlates of lower patient mortality (4-9). In an analysis from the United States Renal Data System (USRDS) Case Mix Adequacy Study, we found that among the four most important predicators of mortality, three were indicators of nutrition (BMI, serum albumin and serum creatinine), with age being the strongest predictor. Comorbid conditions appeared to have a lesser

Address correspondence to: Robert A. Wolfe, PhD, University of Michigan, KECC 315 West Huron, Suite 240, Ann Arbor, Ml 48103. E-mail: usrds@umich.edu

Seminars in Dialysis-Vol 12, Suppl 1 (May-June) 1999 pp. S-29-S-32 predictive power. Figure 1 shows several patient characteristics, ordered by the relative risk (RR) per standard deviation change for each characteristic. The use of RR per standard deviation makes the ordering more meaningful by reflecting the RR for a "typical" change in each factor. The importance of nutritional status emphasizes the fact that single-minded attention to dialysis dose is not sufficient for achieving good patient outcomes.

However, the importance of nutrition should not blind us to the importance of the dose of dialysis. It is well known that it is essential to maintain an adequate dose of dialysis for the ESRD patient, since, without dialysis, death is an early and certain outcome. Several recent observational studies have tried to identify the shape of the relationship of dose of dialysis to mortality and have identified an apparent flattening of the relationship with mortality risk as the dose of dialysis is increased double pool Kt/V (eKt/V) above 1.1 or a single pool Kt/V (spKt/ V) of $1.3(10-12)$. One of the major objectives of the current HEMO trial (13) is the evaluation of the effect of raising the dose of dialysis above the current common practice in the United States.

We strongly suggest that it is crucial to manage both the dose of dialysis and patient nutrition in the treatment of ESRD patients. Here, we examine the links between these two factors, consider in broad terms how dose should be measured, and consider the problem of identifying the optimal dose of dialysis.

\section{Links Between Dose and Nutrition}

Lindsey hypothesized that higher Kt/V would lead to better nutrition in ESRD patients (14). Several observational studies have yielded conflicting data in favor of or against this hypothesis. This hypothesis will be difficult to establish without a clinical trial because dose of dialysis and nutritional status are linked by dialysis practices and by mathematical links between their measures as described below.

The commonly used empirical measures of dose of dialysis and nutritional status are both based on pre- and post-dialysis measures of urea concentration. In practice, $\mathrm{Kt} / \mathrm{V}$ often is not measured through the components, $\mathrm{K}$, $\mathrm{t}$, and $\mathrm{V}$, but is instead inferred based upon measurements of pre- and post-dialysis blood urea nitrogen (BUN) concentrations (15). The PCR is a fundamental measure of protein nutrition, and it is also estimated indirectly through the difference between post- and pre- 


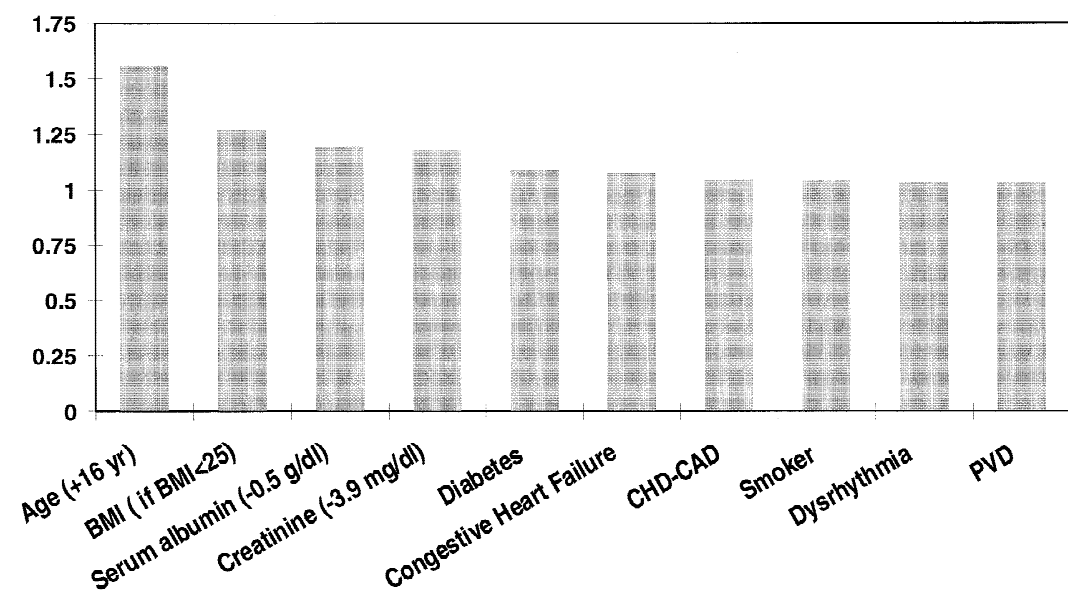

FIG. 1. Mortality RR per 1 SD change in patient characteristics.

dialysis BUN measures. This mathematical link has the potential to cause the observed measured values of $\mathrm{Kt} / \mathrm{V}$ and PCR to be associated, even if there were no association between the true values of these factors, because the errors in measuring BUN are propagated to both $\mathrm{Kt} / \mathrm{V}$ and PCR. This mathematical link, driven by variations (errors) in pre- and post-dialysis BUN measurements, leads to a spurious positive association between the dose of dialysis and PCR.

Higher dose of dialysis is associated with smaller patient volume because of the current dosing practices in the United States $(9,16)$. That is, with current dialysis practice in the United States, Kt is not always prescribed in proportion to patient volume, so that larger patients receive lower values of $\mathrm{Kt} / \mathrm{V}$ on average. This association is seen for other measures related to patient volume, including weight and body mass index (9). This observation leads to a negative association between the dose of dialysis and body size, which is a surrogate for nutritional status and makes it difficult to discern a causal relationship between elevating the dose of dialysis and better nutritional status.

While these links might make it hard to disentangle the separate effects of nutrition and dose on patient mortality, they should not prevent us from recognizing their separate effects nor divert us from trying to separate them empirically. Perhaps the HEMO trial can shed more light on this question.

\section{Measuring Dialysis Dose and the Choice of "Optimal" Dose}

Most standard measures of dose are related to the fractional reduction in pre and post dialysis urea concentrations. Dr. Gotch helped pioneer our understanding of the relationship between the components of dialysis ( $\mathrm{K}$ and t) and urea levels. Kt/ $\mathrm{V}$ is uniquely able to link the prescription of $\mathrm{K}$ and $\mathrm{t}$ to the outcome of reduced urea concentration, depending on the urea distribution space (V).

However, urea reduction is not the ultimate goal of dialysis. Urea is unlikely to be the most important toxin removed by dialysis. However, both empirical associations and logic support the concept that the concentration of urea is a good surrogate for the effects of toxins in the blood (particularly from protein metabolism) and explains the higher mortality seen with lower $\mathrm{Kt} / \mathrm{V}_{\text {urea }}$. Despite its importance in explaining patient mortality, the measurement of $\mathrm{Kt} / \mathrm{V}_{\text {urea }}$ was originally conceived as a measure of dialysis dose, though analyzes by Gotch and Sargent (2) pointed out that it also predicted patient outcomes. There is evidence for a similar relationship that $\mathrm{Kt} / \mathrm{V}$ of middle molecules modifies mortality risk independent of $\mathrm{Kt} / \mathrm{V}_{\text {urea }}$ (17).

Empirical studies have indicated that there may be reduced benefits to elevating the dose of dialysis much above spKt/V = $1.3(\mathrm{eKt} / \mathrm{V}=1.1)$. The optimal dose of dialysis can be defined as being the dose above which little if any improvement in patient outcomes can be observed. Identification of the optimal dose of dialysis, then, is a simple matter of studying the dose response relationship between mortality and dose. Unfortunately, dose-response relationships are difficult to quantitate with the level of precision needed for establishing an optimal dose of dialysis. Specifically, it requires a substantial amount of empirical evidence to identify the dose above which there is no benefit. Further, the relationship of patient size and other characteristics to mortality makes it plausible that this dose may need to be determined separately for different patient subgroups.

Recently, it has been proposed that the optimal dose of dialysis may vary by patient group (18) and can be based on $\mathrm{Kt}$ and gender, rather than upon $\mathrm{Kt} / \mathrm{V}$. As Dr. Lowrie has pointed out, it would not be good medical practice to reduce the level of dialysis delivered to a patient, whose volume decreases due to worsening nutritional status, even though this practice would be a logical approach if $\mathrm{Kt} / \mathrm{V}$ alone were considered as the criterion for choosing the optimal dose of dialysis. Measuring the dose by Kt would not lead to such an inappropriate practice since patients who lose weight (volume) would continue to receive the same level of dialysis. We wholeheartedly agree that $\mathrm{Kt} / \mathrm{V}$ should not be reduced on the basis of reduction in patient volume. However, we are concerned that a failure to account for patient size when choosing the dose of dialysis also ignores the basic relationship, pioneered by Dr. Gotch, between urea concentrations and dose of dialysis. In particular, we hope that the dialysis community does not become so enamored with the ease of prescriptions based on $\mathrm{K}$ and $\mathrm{t}$ alone that the 


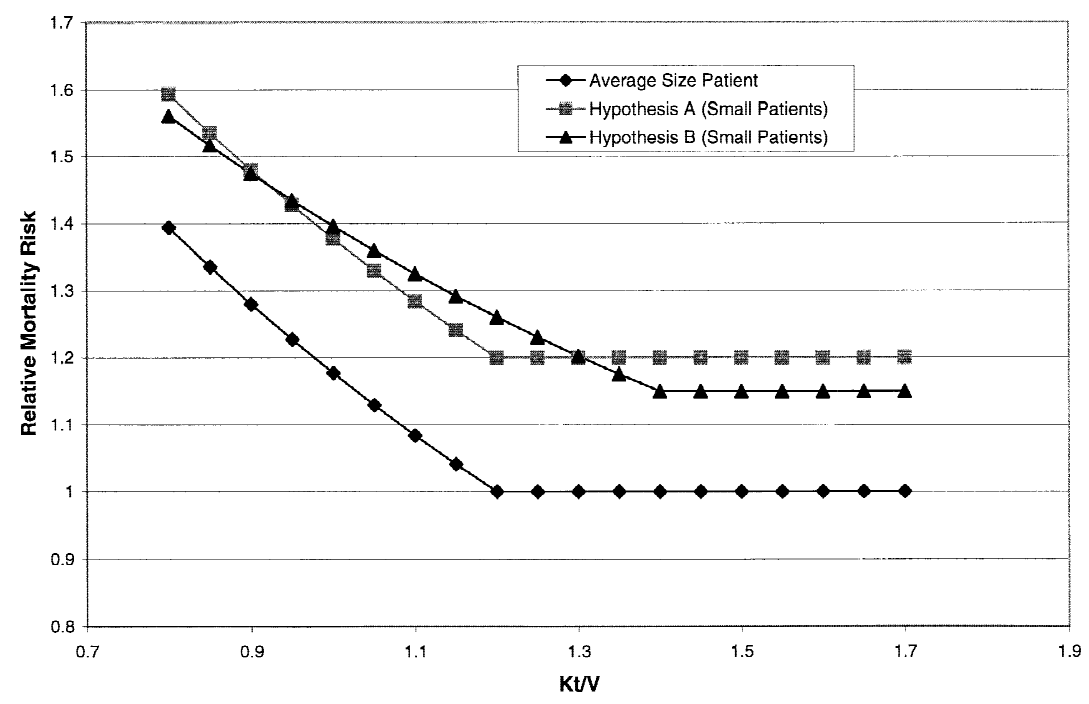

FIG. 2. Hypothetical mortality and dose relationships by size of patient.

measurement of pre- and post-BUN (or other toxin) concentrations is ignored.

There are a variety of approaches to measuring dose that would avoid the problem of reducing the dose for patients whose volume decreases. For example, prescription on the basis of an ideal (rather than current) patient volume may prove to be a useful concept. We should not confuse two different objectives: choosing a good measure for the dose of dialysis, and choosing the optimal level of the dose of dialysis. $\mathrm{Kt} / \mathrm{V}$ is a good measure of the dose of dialysis, despite the fact that it leads to inappropriate dosing practices when patient volume changes. This is akin to recognizing that degrees Kelvin is an excellent measure of temperature despite the fact that it does not explain the boiling point of water at different altitudes.

To reiterate, we believe that the optimal dose of dialysis should be based on empirical evidence. This evidence requires careful calibration of the relationship between dose of dialysis and patient outcomes, especially mortality.

\section{Identification of the Optimal Dose of Dialysis}

It is clear that patient condition, including nutritional status, should be considered when treating ESRD patients. In view of the known association between mortality and patient size, it is plausible that nutrition should be considered when prescribing the dose of dialysis for a patient. We believe that the selection of an optimal dialysis dose is largely an empirical question that does not depend upon whether one uses $\mathrm{Kt} / \mathrm{V}$ or $\mathrm{Kt}$ to measure dose. In fact, for a given patient size (volume), Kt and $\mathrm{Kt} / \mathrm{V}$ are proportional measures of dialysis dose, and it does not matter which is used as the basis for choosing a dose level.

Figure 2 shows the schematic relationship between mortality and $\mathrm{Kt} / \mathrm{V}$ for patients of differing size (nutritional status). The higher mortality shown for smaller patients, particularly lower BMI, has been documented by several studies $(6,9)$. What is not accurately known, is the dose above which the mortality relationship is relatively flat. Hypothesis A shows no survival benefit above a certain $\mathrm{Kt} / \mathrm{V}$ that is independent of patient size, whereas hypothesis B assumes benefits from relatively higher $\mathrm{Kt} / \mathrm{V}$ for smaller with an earlier leveling off for larger patients. It will be difficult to distinguish between the hypothetical relationships A and B shown for small patients, without data for thousands of patients.

The answer to this question can only be based on substantial experience with mortality for a wide variety of types of patients. The USRDS is in a unique position to address this question. Serial measures of the dialysis dose are now being routinely collected for all dialysis patients as requested by HCFA through the ESRD Networks. Follow-up of these patients will allow identification of the relationship between the dose of dialysis and patient outcomes, including both mortality and hospitalization and assessment by patient groups. This level of detailed data can help to answer the specific question by type of patient of how much dialysis is needed to achieve the optimal outcome.

\section{Conclusion}

Clinical experience and logic indicates that both nutritional status and the dose of dialysis, can be modified by the clinical practice independent of one another. In view of their importance to patient survival, both need to be measured in a standardized fashion. Such standardization has been recommended by DOQI (19). Both factors need to be studied to determine levels of optimization while considering other issues such as costeffectiveness.

With the recent addition of the dose of dialysis to the data routinely collected by the HCFA, the USRDS will offer a potential resource for the identification of such optimal doses, for different patient subgroups. This approach is a return to Dr. Gotch's focus on empiricism, which should remain as the highest test of all for our efforts at deductive reasoning.

\section{References}

1. Sargent JA, Gotch FA: Mathematical modeling of dialysis therapy. Kidney Int 18(Suppl):S2-S10, 1976 
2. Gotch F, Sargent JA: A mechanistic analysis of the National Cooperative Dialysis Study (NCDS). Kidney Int 28:526-536, 1985

3. Gotch FA: Kinetic modeling in hemodialysis, in Clinical Dialysis (3rd edition), edited by Nissenson AR, Fine RN, Gentile DE. Englewood Cliffs, NJ, Prentice Hall, 1995, pp 156-189

4. United States Renal Data System: Comorbid conditions and correlations with mortality risk among 3399 incident hemodialysis patients. Am J Kidney Dis 20(Suppl. 2):32-38, 1992

5. Lowrie EG, Lew NL: Death risk in hemodialysis patients: The predictive value of commonly measured variables and an evaluation of death rate differences between facilities. Am J Kidney Dis 15:458-482, 1990

6. Leavey SF, Strawderman RL, Jones CA, Port FK, Held PJ: Simple nutritional indicators as independent predictors of mortality in hemodialysis patients. Am J Kidney Dis 31:997-1006, 1998

7. Port FK: Morbidity and mortality in dialysis patients. Kidney Int 46:17281737, 1994

8. Goldwasser P, Mittman N, Antignani A, Burrell D, Michel M, Collier J, Avram MM: Predictors of mortality in hemodialysis patients. $J$ Am Soc Nephrol 3:1613-1622, 1993

9. Wolfe RA, Ashby V, Hulbert-Shearon T, Agodoa L, Port FK: Body size, dose of hemodialysis and mortality: Results from USRDS Special Study Submitted to Am J Kidney Dis

10. Held PJ, Port FK, Wolfe RA, Stannard DC, Daugirdas JT, Bloembergen WE, Greer JW, Hakim RM: The dose of hemodialysis and patient mortality. Kidney Int 50:550-556, 1996
11. Owen W, Lew N, Liu Y, Lowrie E, Lazarus M: The urea reduction ratio and serum albumin concentration as predictors of mortality in patients undergoing hemodialysis. N Engl J Med 329:1001-1006, 1993

12. Gotch FA, Levin NW, Port FK, Wolfe RA, Uehlinger DE: Clinical outcome relative to the dose of dialysis is not what you think: The fallacy of the mean. Am J Kidney Dis 30:1-15, 1997

13. Eknoyan G, Levey AS, Beck GJ, Agodoa LY, Daugirdas JT, Kusek JW, Levin NW, Schulman G: Hemodialysis (HEMO) study: Rational for selection and interventions. Semin Dial 9:24-33, 1996

14. Lindsay RM, Spanner E: A hypothesis: The protein catabolic rate is dependent upon the type and amount of treatment in dialyzed uremic patients. Am J Kidney Dis 13:382-389, 1989

15. Daugirdas JT: Simplified equations for monitoring Kt/V, PCRn, eKt/V, and ePCRn. Adv Ren Replace Ther 2:295-304, 1995

16. Bloembergen WE, Carroll C, Gillespie B, Young EW, Wolfe RA, Port FK: Why do males with ESRD have higher mortality rates than females? $J$ Am Soc Nephrol 7:1440, 1996

17. Leypoldt JK, Cheung AK, Carroll CE, Stannard DC, Pereira BJG, Agodoa LY, Port FK: Effect of dialysis membranes and middle molecule removal on chronic hemodialysis patient survival. Am J Kidney Dis 33:349-355, 1999

18. Owen WF Jr, Chertow GM, Lazarus JM, Lowrie EG: Dose of hemodialysis and survival: Differences by race and sex. JAMA 280:1764-1768, 1998

19. National Kidney Foundation: NKF-DOQI: Clinical practice guidelines for hemodialysis adequacy. Am J Kidney Dis 30:S15-S66, 1997 